\title{
Effect of screening for type 2 diabetes on risk of cardiovascular disease and mortality: a controlled trial among 139,075 individuals diagnosed with diabetes in Denmark between 2001 and 2009
}

\author{
Rebecca K. Simmons ${ }^{1,2,3,4}$ • Simon J. Griffin ${ }^{1,4,5}$ • Torsten Lauritzen ${ }^{1}$ • Annelli Sandbæk ${ }^{1}$
}

Received: 19 January 2017 / Accepted: 10 April 2017 /Published online: 23 August 2017

(C) The Author(s) 2017. This article is an open access publication

\begin{abstract}
Aims/hypothesis There is continuing debate about the net benefits of population screening for type 2 diabetes. We compared the risk of cardiovascular disease (CVD) and mortality among incident cases of type 2 diabetes in a screened group with those in an unscreened group.

Methods In this register-based non-randomised controlled trial, eligible individuals were all men and women aged 40 69 years without known diabetes, registered with a general practice in Denmark $(n=1,912,392)$. Between 2001 and 2006, 153,107 individuals registered with 181 practices participating in the Anglo-Danish-Dutch Study of Intensive Treatment in People with Screen-Detected Diabetes in Primary Care (ADDITION)-Denmark study were sent a diabetes-risk-score questionnaire. Individuals at moderate-tohigh risk were invited to visit their family doctor for assessment of diabetes status and cardiovascular risk (screening
\end{abstract}

Electronic supplementary material The online version of this article (doi:10.1007/s00125-017-4299-y) contains peer-reviewed but unedited supplementary material, which is available to authorised users.

Rebecca K. Simmons

rks34@cam.ac.uk

1 Department of Public Health, Section of General Practice, Aarhus University, Aarhus, Denmark

2 Danish Diabetes Academy, Odense University Hospital, Odense, Denmark

3 Aarhus Institute of Advanced Studies, Aarhus, Denmark

4 MRC Epidemiology Unit, University of Cambridge School of Clinical Medicine, Box 285 Institute of Metabolic Science, Cambridge Biomedical Campus, Cambridge CB2 0QQ, UK

5 Primary Care Unit, Institute of Public Health, University of Cambridge, Cambridge, UK group). The 1,759,285 individuals registered with all other practices in Denmark constituted the retrospectively constructed no-screening (control) group. In this post hoc analysis, we identified individuals from the screening and noscreening groups who were diagnosed with diabetes between 2001 and $2009(n=139,075)$, and compared risk of CVD and mortality in these groups between 2001 and 2012 .

Results In the screening group, 27,177/153,107 (18\%) individuals attended for screening, of whom 1533 were diagnosed with diabetes. Between 2001 and 2009, 13,992 people were newly diagnosed with diabetes in the screening group (including those diagnosed by screening) and 125,083 in the no-screening group. Between 2001 and 2012, the risks of CVD and mortality were lower among individuals with diabetes in the screening group compared with individuals with diabetes in the noscreening (control) group (CVD HR 0.84, 95\% CI 0.80, 0.89; mortality HR $0.79,95 \%$ CI $0.74,0.84$ ).

Conclusions/interpretation A single round of diabetes screening and cardiovascular risk assessment in middle-aged Danish adults in general practice was associated with a significant reduction in risk of all-cause mortality and CVD events in those diagnosed with diabetes.

Keywords Cardiovascular disease $\cdot$ General practice $\cdot$ Mortality $\cdot$ Population $\cdot$ Screening $\cdot$ Trial $\cdot$ Type 2 diabetes

\begin{tabular}{ll}
\multicolumn{2}{l}{ Abbreviations } \\
ADDITION & $\begin{array}{l}\text { Anglo-Danish-Dutch Study of Intensive } \\
\text { Treatment in People with Screen-Detected } \\
\end{array}$ \\
& $\begin{array}{l}\text { Diabetes in Primary Care } \\
\text { Cardiovascular disease }\end{array}$ \\
CVD & Fasting blood glucose \\
FBG & Ischaemic heart disease \\
IHD & Random blood glucose
\end{tabular}




\section{Introduction}

The potential benefits of screening and early treatment for type 2 diabetes have been widely debated. Modelling studies suggest that screening might be both effective and costeffective [1-5]. Screening and early treatment for diabetes appear to be associated with limited harms [6,7]. Health check programmes including diabetes risk assessment have been proposed or introduced in a number of countries $[8,9]$. However, trials of population-based screening for type 2 diabetes [10] and related cardiovascular risk factors [11] have failed to show significant overall benefit.

While the effect of screening at the population level might be smaller than expected, there may be benefits for those found to have diabetes. Results from the Anglo-DanishDutch Study of Intensive Treatment In People with ScreenDetected Diabetes in Primary Care (ADDITION)-Europe (ClinicalTrials.gov, number NCT00237549) showed that individuals diagnosed with diabetes and treated earlier had a risk of mortality that was similar to that reported for people of the same age without diabetes in the general population in Denmark [12]. One of the challenges of demonstrating potential benefit for those found to have diabetes following screening is that it would not be ethical to conduct a clinical trial of screening and early intervention compared with screening and delayed intervention [13]. As such, we cannot directly observe the magnitude of cardiovascular risk reduction that might occur among individuals with diabetes found by screening compared with those with no screening and hence no treatment until the time of clinical diagnosis [5]. Furthermore, simply comparing screen-detected individuals with clinically diagnosed individuals in a parallel cohort design tends to overestimate benefit because of lead and length time biases.

Between 2001 and 2006, a population-based cardiovascular risk assessment and diabetes screening programme was introduced in general practices in the Danish arm of the ADDITION-Europe study [14]. In the present study, using a controlled design, the Danish national registration system allows us to quantify the extent to which screening brings forward the diagnosis of diabetes, and to conduct a post hoc comparison of the risk of mortality and cardiovascular events among individuals with incident diabetes in the screening group and individuals with incident diabetes in the noscreening (control) group.

\section{Methods}

ADDITION-Europe is a cluster-randomised trial comparing the effects of screening for type 2 diabetes followed by intensive multifactorial therapy of individuals with screen-detected diabetes and screening followed by routine care $[14,15]$. We report results from a post hoc analysis using data from the screening phase of the Danish arm of the study in conjunction with data from Danish national registers. Ethical approval for the ADDITION-Denmark study was granted by a local scientific committee (number 20000183). As this was a registry-based study using anonymised data, participants did not give informed consent. This approach was approved by the Danish Data Protection Agency and the Danish Health and Medicine Authority.

Screening programme Full details of the programme have been reported $[11,12,14]$. In brief, we performed a population-based stepwise screening programme in people aged 40 to 69 years, without known diabetes, between 2001 and 2006 [14-16]. All general practices in five out of 16 counties in Denmark (Copenhagen, Aarhus, Ringkoebing, Ribe and South Jutland) were invited to take part in ADDITION-Denmark ( $n=744) ; 209$ (28.1\%) accepted.

Eligible individuals registered with the 181 practices who agreed to take part were sent a diabetes-risk-score questionnaire, $[15,16]$ with an invitation to visit their general practitioners for a diabetes test and a cardiovascular risk assessment if they scored $\geq 5$ (maximum 15) points or were invited when visiting the practice for another reason ( $n=35$ practices). No reminders were sent. Participants who attended a screening appointment underwent measurement of height, weight and blood pressure. A capillary blood sample was taken for testing of random blood glucose (RBG). A venous blood sample was taken for measurement of total cholesterol and $\mathrm{HbA}_{1 \mathrm{c}}$. General practitioners were encouraged to calculate the European Heart SCORE [17] during the appointment, to inform individuals about their score and provide appropriate advice and treatment to those at high risk. Individuals with an $\mathrm{RBG} \geq 5.5 \mathrm{mmol} / \mathrm{l}$ or $\mathrm{HbA}_{1 \mathrm{c}} \geq 5.8 \%$ were invited to return to the practice for a fasting blood glucose (FBG) (capillary) test. An OGTT was performed at the same consultation if FBG was 5.6-6.1 mmol/l and/or $\mathrm{HbA}_{1 \mathrm{c}} \geq 5.8 \%$. WHO 1999 criteria were used to diagnose diabetes [18], including the requirement for a confirmatory test on another day.

In the screening group, participants diagnosed with type 2 diabetes were subsequently managed according to the treatment regimen to which their practice was allocated: routine care or intensive treatment [12].

Sampling frame We identified all eligible individuals in the original ADDITION-Denmark study $(n=153,107)$, including those who did not attend for screening, on the Danish National Registry system (the screening group). Using the same registry, we also identified all individuals aged 40-69 years without known diabetes who, between 2001 and 2006, were registered with general practices that were not invited to take part in ADDITION-Denmark or who declined to take part in ADDITION-Denmark ( $n=1,759,285)$ (the no-screening control group). We then identified individuals from the screening 
and no-screening groups who were diagnosed with incident diabetes between 1 January 2001 and 31 December 2009 (Fig. 1). We included individuals diagnosed with diabetes during this period based on recent estimates of lead time, which suggest that there is around 3 years between detection by screening and clinical diagnosis. Including individuals diagnosed with diabetes in the 3 years (2006-2009) following the end of the ADDITION screening phase (2001-2006) would therefore capture most individuals in the no-screening group who could have been diagnosed by screening if they had been in the screening group. Our definition of incident diabetes for both groups was a proxy measure based on date of inclusion in the Danish National Diabetes Register [19]. We linked information about individuals diagnosed with diabetes to other Danish registers using unique civil registration numbers. We retrieved information on age, sex, education, immigration/emigration, citizenship, redeemed cardioprotective medication and chronic disease. Education was categorised according to Unesco's International Standard Classification of Education [20]. We grouped data on citizenship into European and nonEuropean as a proxy for ethnicity.

Outcomes Participants were followed for a median of 6.2 years until 31 December 2012, when national registers were searched for information on vital status and incident cardiovascular disease (CVD) events. For death, the primary outcome was all-cause mortality (based on underlying cause of death). Secondary outcomes were cardiovascular-, cancer- and diabetes-related mortality. Cause-specific deaths were coded blind to study group using ICD-10 codes (www.who.int/classifications/icd/en/; electronic supplementary material [ESM] Table 1). For CVD, the primary outcome was a composite of first event of cardiovascular death, non-fatal IHD (ICD-10 codes I20-I25, I46) or nonfatal stroke (ICD-10 code I6*). Data were gathered from the National Patient Registry, which records all inpatient and outpatient hospitalisations in Denmark.

Statistical analysis We summarised the characteristics of all individuals diagnosed with incident diabetes between 2001 and 2009 separately in the screening and no-screening groups. Date of entry to the study was the date of inclusion on the diabetes register. Individuals were censored on the date of first event, date of emigration or 31 December 2012, whichever was earliest. We estimated HRs comparing mortality outcomes and incident CVD in people diagnosed with diabetes in the screening and noscreening groups with a Cox proportional hazards regression model. As allocation to screening and no-screening groups was at the practice level, robust standard errors were calculated that take into account the two-level structure of the data [21]. We adjusted for age, sex, education and prevalent chronic disease (ischaemic heart disease [IHD], stroke, cancer) at baseline. To account for differences in social structure, we stratified the baseline hazards by county. We conducted a subgroup analysis, rerunning the all-cause mortality model separately by age ( $<55$ years or $\geq 55$ years) and sex. To calculate the proportion of individuals redeeming cardioprotective medication in each calendar year, we included all individuals who were alive on 31 December of the year in question and who had previously been diagnosed with diabetes. All analyses were completed using Stata Version 14.1 (STATA, College Station, TX, USA). Statistical significance was inferred at a two-tailed $p<0 \cdot 05$.
Fig. 1 Visual representation of sampling frame. The ' $\mathrm{S}$ ' in a blue circle denotes individuals detected by the ADDITION stepwise screening programme. The ' $\mathrm{C}$ ' in a red circle denotes individuals with clinically diagnosed diabetes

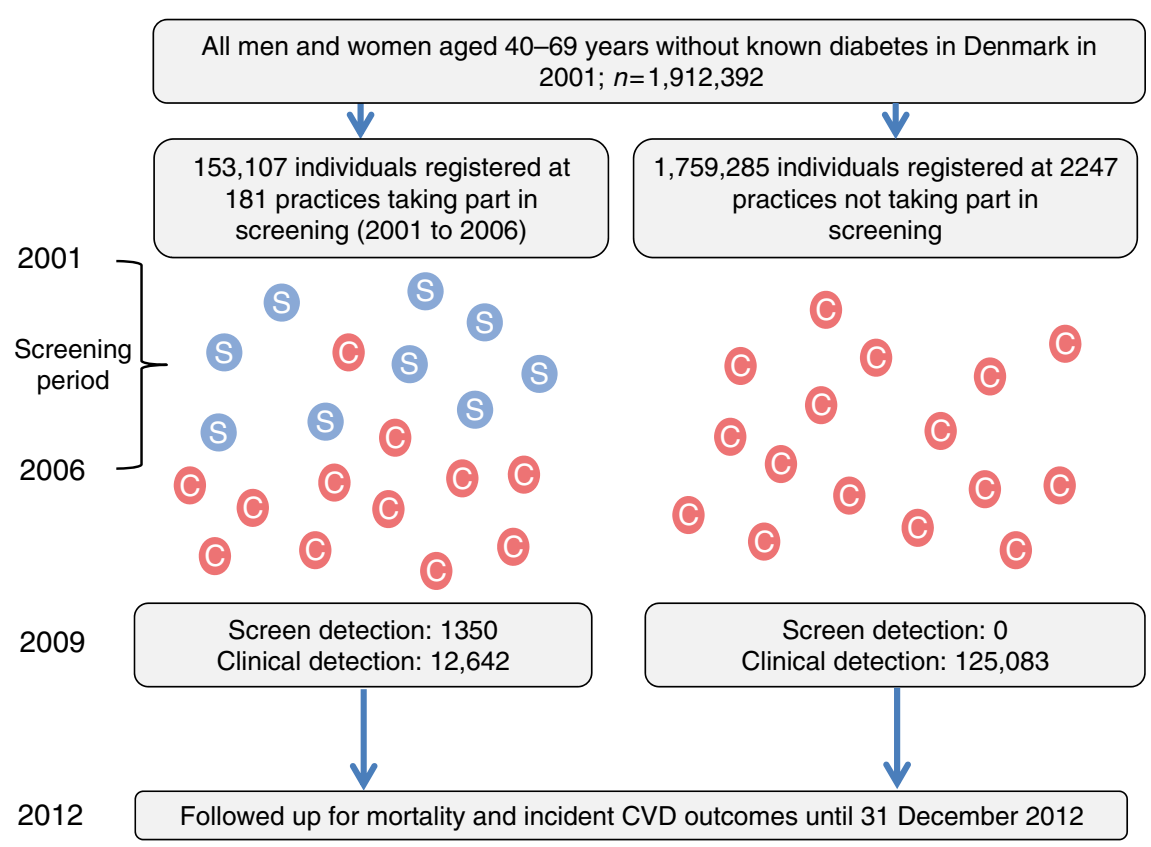


Table 1 Characteristics of individuals with diabetes by screening group

\begin{tabular}{lcc}
\hline Characteristic & $\begin{array}{l}\text { Screening group } \\
n=13,992\end{array}$ & No-screening (control) group $n=125,083$ \\
\hline Mean age at diagnosis (SD), years & $59.9(7.7)$ & $59.2(9.2)$ \\
Male sex, $n(\%)$ & $7495(53.6)$ & $70,559(56.4)$ \\
Years of education, n (\%) & & \\
$\quad 0$ to 10 & $5610(40.1)$ & $55,770(44.6)$ \\
10 to 15 & $6237(44.6)$ & $55,230(44.2)$ \\
$\quad 15+$ & $2145(15.3)$ & $14,083(11.3)$ \\
European citizenship, $n(\%)^{\mathrm{a}}$ & $13,809(99.0)$ & $121,572(98.2)$ \\
Previous IHD, $n(\%)^{\mathrm{b}}$ & $1586(11.3)$ & $16,217(13.0)$ \\
Previous stroke, $n(\%)^{\mathrm{b}}$ & $628(4.5)$ & $6852(5.5)$ \\
Previous cancer, $n(\%)^{\mathrm{b}}$ & $2027(14.5)$ & $19,276(15.4)$ \\
\hline
\end{tabular}

${ }^{a}$ Totals do not match denominator owing to missing data

${ }^{\mathrm{b}}$ Data taken from the National Patient Registry; data included from 1994 until date of diabetes diagnosis

\section{Results}

Of 153,107 eligible people in the screening group, $27,177(18 \%)$ attended their general practice for a diabetes test and cardiovascular risk assessment. Of these, 1533 participants (1\% of those eligible for screening) were diagnosed with diabetes and agreed to take part in the study; 1406 of these were subsequently included on the diabetes register. There were 1,759,285 individuals in the no-screening group.

Between 1 January 2001 and 31 December 2009, 139,075 people from our sampling frame were diagnosed with incident diabetes and included on the Danish National Diabetes Register. Of these, $13,992(10.1 \%)$ were in the screening group and 125,083 (89.9\%) in the no-screening group. There were 83,385 (60.5\%) clinically diagnosed cases of diabetes detected during the screening period (2001 to 2006) and 54,340 (39.5\%) cases between 2007 and 2009. The groups were well balanced for age and citizenship (Table 1). There were slightly more men in the no-screening group (56.4\%) compared with the screening group (53.6\%). Compared with the no-screening group, a larger proportion of the screening group had received $15+$ years of education. Slightly higher proportions of the no-screening group had experienced IHD, stroke or cancer.

Median diabetes duration among clinically diagnosed individuals in the screening group was 6.6 years (interquartile range [IQR] 4.6 to 9.4 ) compared with 8.8 years (IQR 6.9 to

Table 2 Redeemed cardioprotective medication from 2001 to 2009 by screening group

\begin{tabular}{|c|c|c|c|c|c|c|c|c|c|c|}
\hline \multirow[t]{3}{*}{ Year } & \multicolumn{3}{|c|}{ Glucose-lowering medication $^{\mathrm{a}}$} & \multicolumn{3}{|c|}{ Lipid-lowering medication ${ }^{\mathrm{a}}$} & \multicolumn{3}{|c|}{ Anti-hypertensive medication $^{\mathrm{a}}$} & \multirow[t]{3}{*}{ Total $^{\mathrm{b}}$} \\
\hline & \multirow{2}{*}{$\begin{array}{l}\text { No-screening } \\
\text { group }\end{array}$} & \multicolumn{2}{|c|}{ Screening group } & \multirow{2}{*}{$\begin{array}{l}\text { No-screening } \\
\text { group }\end{array}$} & \multicolumn{2}{|c|}{ Screening group } & \multirow{2}{*}{$\begin{array}{l}\text { No-screening } \\
\text { group }\end{array}$} & \multicolumn{2}{|c|}{ Screening group } & \\
\hline & & $\begin{array}{l}\text { Clinically } \\
\text { diagnosed }\end{array}$ & $\begin{array}{l}\text { Screen- } \\
\text { detected }\end{array}$ & & $\begin{array}{l}\text { Clinically } \\
\text { diagnosed }\end{array}$ & $\begin{array}{l}\text { Screen- } \\
\text { detected }\end{array}$ & & $\begin{array}{l}\text { Clinically } \\
\text { diagnosed }\end{array}$ & $\begin{array}{l}\text { Screen- } \\
\text { detected }\end{array}$ & \\
\hline 2001 & $4928(49.9)$ & $334(31.4)$ & $31(20.8)$ & $1518(15.4)$ & $141(13.2)$ & $26(17.5)$ & $5396(54.6)$ & $543(51.0)$ & $77(51.7)$ & 11,097 \\
\hline 2002 & $10,028(48.2)$ & $641(25.0)$ & $106(25.9)$ & $4679(22.5)$ & $454(17.7)$ & $132(32.2)$ & $11,879(57.1)$ & $1289(50.3)$ & $250(61.0)$ & 23,777 \\
\hline 2003 & $16,288(49.2)$ & $1050(25.8)$ & $240(34.7)$ & $10,703(32.3)$ & $1098(27.0)$ & $330(47.8)$ & $19,823(59.9)$ & $2183(53.7)$ & 459 (66.4) & 37,866 \\
\hline 2004 & $23,401(50.8)$ & $1461(28.9)$ & $373(39.4)$ & $18,936(41.1)$ & $1812(35.8)$ & $585(61.8)$ & $29,043(63.0)$ & $2913(57.5)$ & $664(70.2)$ & 52,092 \\
\hline 2005 & $30,458(52.5)$ & $1900(31.0)$ & $464(43.4)$ & $27,176(46.9)$ & $2539(41.4)$ & $712(67.4)$ & $38,124(65.8)$ & $3707(60.4)$ & 787 (74.5) & 65,180 \\
\hline 2006 & $38,453(54.8)$ & $2412(31.2)$ & $583(47.1)$ & $37,091(52.9)$ & $3536(45.7)$ & $920(74.3)$ & $47,938(68.3)$ & $4854(62.8)$ & 948 (76.5) & 79,137 \\
\hline 2007 & $47,162(56.5)$ & $2986(32.9)$ & $663(52.7)$ & $48,218(57.7)$ & 4689 (51.7) & 974 (77.4) & $58,837(70.4)$ & 5967 (65.8) & 1003 (79.9) & 93,851 \\
\hline 2008 & $57,087(58.0)$ & $3618(34.3)$ & $737(58.5)$ & $60,981(62.0)$ & $5895(55.9)$ & $1018(80.8)$ & $71,373(72.5)$ & 7213 (68.4) & $1035(82.1)$ & 110,198 \\
\hline 2009 & $67,071(59.2)$ & $4292(36.6)$ & 764 (60.9) & $71,654(63.3)$ & $6763(57.7)$ & $1016(81.0)$ & $83,834(74.0)$ & 8273 (70.6) & 1048 (83.6) & 126,225 \\
\hline
\end{tabular}

Parentheses show the proportion (\%) of individuals in each screening group who redeemed a particular class of cardio-protective medication in each year ${ }^{a}$ Anatomical Therapeutic Chemical (ATC) codes: glucose-lowering medication (A*); lipid-lowering medication (C10*); anti-hypertensive medication $\left(\mathrm{CO} 7 *, \mathrm{CO}^{*}, \mathrm{C} 09^{*}\right)$

${ }^{\mathrm{b}}$ Based on all individuals alive on 31 December of the year in question who had previously been diagnosed with diabetes 
10.1) in screen-detected individuals in the screening group (difference 2.2 years, $p<0.001$ ).

In the first year of follow-up, there were 11,097 cases of incident diabetes. Among these individuals, larger numbers of people in the no-screening group redeemed glucose-lowering medication $(49.9 \%)$ compared with clinically diagnosed $(31.4 \%)$ and screen-detected individuals $(20.8 \%)$ in the screening group (Table 2). This difference persisted for clinically diagnosed individuals in the screening and no-screening groups throughout follow-up. However, there was a steep increase in the proportion of screen-detected individuals redeeming glucose-lowering medication, rising from $20.8 \%$ in 2001 to $60.9 \%$ in 2009 . Slightly higher numbers of clinically diagnosed individuals from the no-screening group redeemed lipid-lowering and anti-hypertensive treatment compared with clinically diagnosed individuals in the screening group throughout follow-up (difference between 3\% and 5\%). For individuals with screen-detected diabetes, we observed very large increases in lipid-lowering and anti-hypertensive treatment, rising from $17.5 \%$ and $51.7 \%$ in 2001 , to $81.0 \%$ and $83.6 \%$ in 2009 , respectively. Overall, this group redeemed the highest proportion of all cardioprotective medication by the end of follow-up.

Median duration of follow-up was 6.2 years (IQR 4.2 to 8.8), with 898,285 person-years of observation. During follow-up, there were 1775 deaths in people with diabetes in the screening group $(12.7 \%)$ and 19,739 deaths in people with diabetes in the no-screening group $(15.8 \%)$ (Table 3$)$. The HR for mortality was $0.79,95 \%$ CI $0.74,0.84, p<0.0001$ (Fig. 2). The most common cause of death was cancer $(n=10,500 ; 38.2 \%)$. In subgroup analyses, the HR for mortality was similar among individuals aged $<55$ years and $\geq 55$ years (HR $0.81,95 \%$ CI 0.71 , 0.93, and HR $0.78,95 \%$ CI $0.73,0.84$, respectively). The HRs for mortality were $0.82(95 \%$ CI $0.76,0.88)$ in men and 0.75 $(95 \%$ CI $0.68,0.82)$ in women.

Cardiovascular mortality and cancer mortality were both significantly lower in the screening group compared with the no-screening group (HR 0.80, 95\% CI 0.72, 0.88 and HR $0.83,95 \%$ CI $0.77,0.89$, respectively). Diabetes was listed anywhere on the death certificate in 1822 individuals (121 in the screening group and 1701 in no-screening group), with a significant difference between groups in diabetes-related mortality (HR $0.66,95 \%$ CI $0.54,0.81$ ).

There were 2854 first CVD events among people with diabetes in the screening group (20.4\%) and 28,487 first CVD events among people with diabetes in the no-screening group (22.8\%) (Table 3), with a significant difference between groups (HR 0.84, 95\% CI 0.80, 0.89) (Fig. 3).

\section{Discussion}

In this very large population-based sample of middle-aged Danish adults with 898,285 person-years of follow-up, a single round of diabetes screening and cardiovascular risk assessment was associated with a $21 \%$ reduction in all-cause mortality rate and a $16 \%$ reduction in CVD events between 2001 and 2012 in individuals diagnosed with diabetes between 2001 and 2009. Individuals with clinically diagnosed diabetes were identified on average 2.2 years later than individuals with diabetes detected by screening.

One argument for considering screening for type 2 diabetes is the historical observation [22] that there is an extended latent or pre-clinical phase (lead time) in which people could be diagnosed and during which earlier treatment might have a beneficial longterm effect. More recently, changes in clinical practice with

Table 3 Incidence of all-cause, cardiovascular-, cancer- and diabetes-related mortality, and CVD events, in individuals with diabetes, by screening group (2001 to 2012)

\begin{tabular}{|c|c|c|c|c|c|c|c|c|}
\hline \multirow[t]{2}{*}{ Variable } & \multicolumn{3}{|c|}{ Screening group $(n=13,992)$} & \multicolumn{3}{|c|}{ No-screening group $(n=125,083)$} & \multirow{2}{*}{$\begin{array}{l}\text { Crude HR } \\
(95 \% \mathrm{CI})\end{array}$} & \multirow{2}{*}{$\begin{array}{l}\text { Adjusted } \mathrm{HR}^{\mathrm{a}} \\
(95 \% \mathrm{CI})\end{array}$} \\
\hline & $\begin{array}{l}\text { Number } \\
\text { of } \\
\text { events }\end{array}$ & $\begin{array}{l}\text { Person- } \\
\text { years } \\
\text { follow- } \\
\text { up }\end{array}$ & $\begin{array}{l}\text { Rate per } 1000 \\
\text { person-years } \\
(95 \% \mathrm{CI})\end{array}$ & $\begin{array}{l}\text { Number } \\
\text { of } \\
\text { events }\end{array}$ & $\begin{array}{l}\text { Person- } \\
\text { years } \\
\text { follow- } \\
\text { up }\end{array}$ & $\begin{array}{l}\text { Rate per } 1000 \\
\text { person-years } \\
(95 \% \mathrm{CI})\end{array}$ & & \\
\hline All-cause mortality & 1775 & 97,265 & $18.2(17.4,19.1)$ & 19,739 & 801,019 & $24.6(24.3,25.0)$ & $0.71(0.68,0.75)$ & $0.79(0.74,0.84)$ \\
\hline Cardiovascular mortality & 509 & 97,265 & $5.2(4.8,5.7)$ & 5835 & 801,019 & $7.3(7.1,7.5)$ & $0.70(0.64,0.77)$ & $0.80(0.72,0.88)$ \\
\hline Cancer mortality & 954 & 97,265 & $9.8(9.2,10.5)$ & 9546 & 801,019 & $11.9(11.7,12.2)$ & $0.79(0.74,0.84)$ & $0.83(0.77,0.89)$ \\
\hline Diabetes-related mortality & 121 & 97,265 & $1.2(1.0,1.5)$ & 1701 & 801,019 & $2.1(2.0,2.2)$ & $0.57(0.48,0.69)$ & $0.66(0.54,0.81)$ \\
\hline $\begin{array}{l}\text { Composite cardiovascular event } \\
\text { (first of CVD death, non-fatal } \\
\text { IHD or non-fatal stroke) }\end{array}$ & 2854 & 86,317 & $33.1(31.9,34.3)$ & 28,487 & 698,893 & $40.8(40.3,41.2)$ & $0.78(0.75,0.81)$ & $0.84(0.80,0.89)$ \\
\hline
\end{tabular}

\footnotetext{
${ }^{\text {a }}$ HRs were estimated with a Cox proportional hazards regression model. Robust standard errors were calculated that take into account the two-level structure of the data and any potential correlation between individuals within practices

Models were adjusted for age, sex, education and prevalent chronic disease (IHD, stroke, cancer); baseline hazards were stratified by county
} 


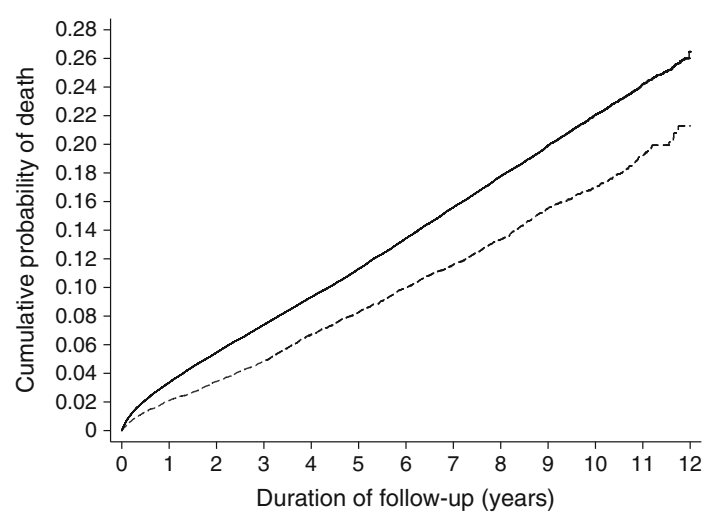

Fig. 2 Cumulative incidence of all-cause mortality in individuals with diabetes in the screening and no-screening groups (2001 to 2012). The model is unadjusted. Solid line, no-screening (control) group; dashed line, screening group

greater testing and public awareness have probably led to a shortening of this latent period. Data from the parallel-group population-based Ely study suggested that the lead time is relatively short at 3.3 years [23]. This is comparable with our more contemporary estimate of 2.2 years. However, the lead time may be longer in less developed health systems and/or in more deprived populations. In addition, the historical estimate of 9 to 12 years by Harris et al. [22] and the more recent estimate of 6 years from Porta et al. [24] relates to the true point of onset of diabetes. This is not the same as the point at which diabetes is detectable by screening, especially if screening is infrequent and not $100 \%$ sensitive. The period between true onset and clinical diagnosis of diabetes may be long precisely because there are few clinical manifestations during this period [23].

Even with a relatively modest lead time, our screening programme was associated with a significant reduction in mortality and incident CVD in individuals with diabetes over 6 years of follow-up. As only $10.1 \%$ of individuals in the screening group were actually diagnosed by screening, it is likely that the programme had wider effects in this cohort e.g. by delaying diagnosis and providing lifestyle advice (and

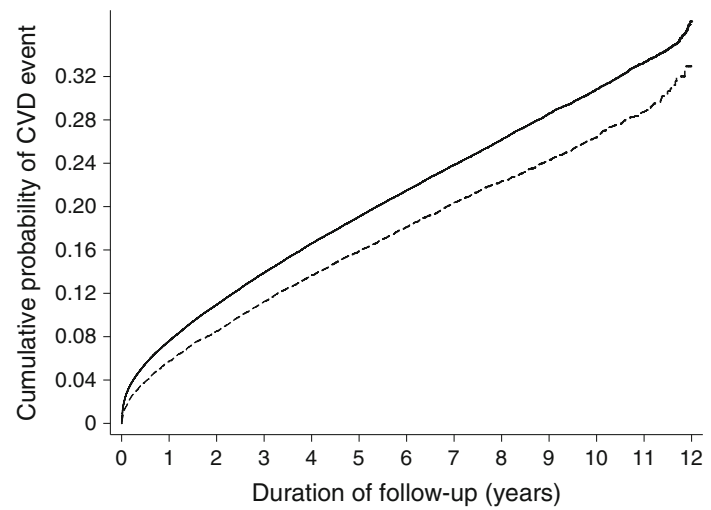

Fig. 3 Cumulative incidence of a composite CVD event among individuals with diabetes in the screening and no-screening groups (2001 to 2012). The model is unadjusted. Solid line, no-screening (control) group; dashed line, screening group perhaps treatment) among those screened and found to be at risk who were later diagnosed clinically. The difference in mortality and CVD might also have been driven by screening practices that were vigilant for diabetes even after the programme had finished, contributing to continued earlier detection and the higher diabetes incidence observed in screening compared with no-screening practices.

Our findings support results from modelling studies showing that screening programmes could contribute to a reduction in risk of mortality and cardiovascular morbidity in screen-detected individuals [1-5]. Herman et al. used a validated computer simulation model in ADDITION-Europe to show that screening and routine care, compared with a 3 year delay in diagnosis and routine care, was associated with a $17 \%$ relative risk reduction in all-cause mortality after 5 years [5]. They argue that the benefits of screening and treatment primarily accrue from early diagnosis and by hastening the treatment of CVD risk factors in the lead time [5]. We observed a rapid increase in the proportion of screen-detected individuals who redeemed cardioprotective medication during follow-up. However, larger proportions of clinically diagnosed individuals in the no-screening group redeemed medication compared with clinically diagnosed individuals in the screening group. As individuals in the no-screening group were diagnosed at a later stage in the disease trajectory, they may have had higher cholesterol, blood glucose and blood pressure values at diagnosis compared with the screening group, necessitating higher levels of cardioprotective medication. It is likely that promotion of healthy behaviour change also impacted on CVD and mortality rates in the screening group. Those who attended screening reported their smoking status at baseline (28\%), which was similar to national self-reported smoking prevalence data in 2004 (Danish National Health Service survey) [25]. One-third of screen-detected individuals in ADDITIONDenmark reported that they had stopped smoking at 5 year follow-up. Furthermore, this cohort lost an average of $2 \mathrm{~kg}$ in weight [12]. If similar behavioural responses were observed among other individuals diagnosed with diabetes in the screening group, this suggests potential mechanisms for the risk reduction observed other than prescribed treatment. We also observed lower rates of cancer incidence in the screening group, which might be linked to changes in health behaviour and prescribing [26].

In a separate paper [11], we examine the impact of the ADDITION-Denmark screening programme at the population level, e.g. comparing all individuals aged 40 to 69 years in the screening and no-screening groups, and showed no long-term reduction in mortality or CVD. As such, our results mirror those from trials of screening for other conditions, which have shown reductions in disease-specific mortality but not in overall mortality [27]. There appeared to be beneficial effects for all those diagnosed with diabetes in the screening practices, regardless of the mode of diagnosis e.g. by screen detection or by clinical diagnosis. However, this benefit is too small to impact on overall population risk of CVD events and mortality $[10,11]$. 
Strengths and limitations This very large controlled trial with long-term follow-up included all individuals aged 40 to 69 years diagnosed with diabetes in Denmark between 2001 and 2009. Outcome ascertainment was robust. The National Death Registry estimates $100 \%$ coverage of mortality based on death certificates. All-cause mortality is an all-inclusive measure that addresses both direct and indirect effects of screening, and puts disease-specific mortality reduction in the context of other competing risks [27]. We were able to ascertain which individuals were living in Denmark in 2001 and censor those who emigrated during follow-up. Deaths and CVD events were coded blind to study group.

Our definition of clinically diagnosed diabetes was a proxy measure based on date of inclusion in the Danish National Diabetes Register, where individuals are classified as having diabetes according to a number of criteria [19, 28]. Using registry-defined diabetes ensures that the entire Danish population is covered by uniform inclusion criteria and the dropout rate is nil. However, we did not have formal clinical diagnosis of diabetes or the date of diagnosis. A recent validation of the algorithm for including individuals in the Register suggests that it has a sensitivity $\geq 95 \%$ and a positive predictive value of around $80 \%$ [19]. The same report also suggests that around $20 \%$ of diabetes diagnoses in the Register may represent false-positive inclusions of people with frequent measurements of blood glucose who do not have diabetes. This may help account for the higher incidence of diabetes in the screening group and help explain the lower levels of cardioprotective medication redeemed by clinically diagnosed individuals in the screening group. Many high-risk individuals would have undergone frequent measurements of blood glucose during the screening programme, delineating them with a diabetes diagnosis on the Register, when they did not in fact have diabetes and were therefore unlikely to receive cardioprotective treatment.

In total, 1406/1533 (92\%) of individuals diagnosed with screen-detected diabetes in ADDITION-Denmark were added to the Diabetes Register with a median delay of 56 days (R. K. Simmons, unpublished data). While this proxy date means our estimate of diabetes duration is probably shorter than the actual length, this is unlikely to be differential by group. By only including individuals aged 40 to 69 years in our study, we assume the number of clinically diagnosed type 1 diabetes cases is likely to be low and similar in both groups.

A limitation of our study is the non-randomised design; we cannot eliminate the possibility of selection bias and residual confounding. Groups were well balanced for most characteristics at baseline. However, our findings might have been influenced by the higher levels of education and the slightly lower levels of pre-existing chronic disease in the screening group. We did adjust for age, sex, education and prevalent chronic disease, which had a small impact on the effect size. Including adjustment by county had a large impact, reducing the effect size considerably, though the hazard remained significant. It is likely that adjusting for county took account of some of the potential socioeconomic differences across different regions in Denmark.

We tried to minimise lead and length time biases by comparing outcomes for all individuals diagnosed with diabetes in the screening and no-screening groups. Further, we extended the inclusion period to 3 years beyond the end of the formal screening programme so that people who could have been detected by screening (had they been in the screening group) were included. However, the small difference in the overall incidence between the groups suggests that some of the observed effect may be due to residual lead and length time bias. Participation in the programme may also have impacted on subsequent diabetes detection rates in screening practices.

While we were able to compare trends in redeemed cardioprotective medication to explore a potential mechanism for the observed difference in outcomes, we did not have populationlevel data on dietary, physical activity or smoking behaviour. The majority of participants were white, the main ethnic group in Denmark, which also limits generalisability to other settings.

In conclusion, a single round of diabetes screening and cardiovascular risk assessment in middle-aged Danish adults performed in general practice was associated with a $21 \%$ reduction in all-cause mortality rates and a $16 \%$ reduction in CVD events between 2001 and 2012 among people diagnosed with diabetes between 2001 and 2009. Screening resulted in cases being identified, on average, 2.2 years earlier.

Acknowledgements We gratefully acknowledge the contribution of all participants, practice nurses and general practitioners in the ADDITIONDenmark study. With special thanks to M. Pedersen (Department of Public Health, University of Aarhus) for her help retrieving data from Statistics Denmark.

Data availability The data used in this paper are a combination of data obtained from the ADDITION study and data from the national Danish Registers at Statistics Denmark (DST). The ADDITION study data were obtained after a written application to the data owner, A. Sandbæk (Institute of Public Health, Aarhus University, Aarhus, Denmark; annelli.sandbaek@ph.au.dk). Data from DST were made available following an application to DST (www.dst.dk/en/TilSalg/ Forskningsservice). Data from ADDITION and DST were merged by DST and analyses were performed via a secure virtual private network (VPN) connection. In accordance with the Danish Act on Processing of Personal Data, future interested researchers must perform the steps mentioned above to obtain access to the data.

Funding ADDITION-Denmark was supported by the National Health Services in the counties of Copenhagen, Aarhus, Ringkøbing, Ribe and South Jutland in Denmark, the Danish Council for Strategic Research, the Danish Research Foundation for General Practice, the Novo Nordisk Foundation, the Danish Centre for Evaluation and Health Technology Assessment, the diabetes fund of the National Board of Health, the Danish Medical Research Council and the Aarhus University Research Foundation. The trial has been supported by unrestricted grants from Novo Nordisk A/S, Novo Nordisk Scandinavia AB, Novo Nordisk UK, ASTRA Denmark, Pfizer Denmark, GlaxoSmithKline Pharma Denmark, Servier Denmark A/S and HemoCue Denmark A/S. 
Duality of interest RKS is supported by the Danish Diabetes Academy under a Visiting Professorship. The Danish Diabetes Academy is funded by the Novo Nordisk Foundation. RKS is further supported by the Aarhus Institute of Advanced Studies. SJG's research programme is supported by MRC Epidemiology Unit core funding (MC UU 12015/4). SJG is an NIHR Senior Investigator and a member of the NIHR School for Primary Care Research. SJG receives an honorarium and reimbursement of travel expenses from Eli Lilly associated with membership of an independent data monitoring committee for a randomised trial of a medication to lower glucose. SJG received an honorarium from Janssen for speaking at an educational meeting in 2015. TL holds shares in Novo Nordisk A/S. TL reports receiving a fee for attending an international board meeting for Astra Zeneca on early detection and treatment of diabetes in 2015. AS reports receiving lecture fees for providing continuing medical education to general practitioners.

Contribution statement RKS and AS had full access to all of the data in the study and take responsibility for the integrity of the data and the accuracy of the data analysis. RKS conducted and is responsible for the data analysis.

RKS and AS act as guarantors for this paper. AS and TL designed the ADDITION-Denmark study, and are principal investigators for the trial. SJG conceived the study question for this paper. SJG, TL, AS and RKS developed the study proposal. AS and RKS participated in the acquisition of the data from Statistics Denmark. RKS analysed the data and drafted the report. RKS, SJG, TL and AS participated in the interpretation of the data, the critical revision of the report for important intellectual content and gave final approval for this version to be published. RKS, TL and AS provided administrative, technical and material support for the study.

Open Access This article is distributed under the terms of the Creative Commons Attribution 4.0 International License (http:// creativecommons.org/licenses/by/4.0/), which permits unrestricted use, distribution, and reproduction in any medium, provided you give appropriate credit to the original author(s) and the source, provide a link to the Creative Commons license, and indicate if changes were made.

\section{References}

1. Kuo HS, Chang HJ, Chou P, Teng L, Chen TH (1999) A Markov chain model to assess the efficacy of screening for non-insulin dependent diabetes mellitus (NIDDM). Int J Epidemiol 28:233-240

2. Chang HJ, Kuo HS, Tung TH, Chou P, Chen TH (2000) Evaluation of a population-based screening for type 2 diabetes: a communitybased screening project in Puli, Taiwan. Prev Med 31:396-402

3. Kahn R, Alperin P, Eddy D et al (2010) Age at initiation and frequency of screening to detect type 2 diabetes: a cost-effectiveness analysis. Lancet 375:1365-1374

4. Schuetz CA, Alperin P, Guda S et al (2013) A standardized vascular disease health check in europe: a cost-effectiveness analysis. PLoS One 8:e66454

5. Herman WH, Ye W, Griffin SJ et al (2015) Early detection and treatment of type 2 diabetes reduce cardiovascular morbidity and mortality: a simulation of the results of the Anglo-Danish-Dutch study of intensive treatment in people with screen-detected diabetes in primary care (ADDITION-Europe). Diabetes Care 38:1449-1455

6. Eborall HC, Griffin SJ, Prevost AT, Kinmonth AL, French DP, Sutton S (2007) Psychological impact of screening for type 2 diabetes: controlled trial and comparative study embedded in the ADDITION (Cambridge) randomised controlled trial. BMJ 335:486

7. Adriaanse MC, Snoek FJ (2006) The psychological impact of screening for type 2 diabetes. Diabetes Metab Res Rev 22:20-25

8. Department of Health (2008) Putting prevention first. Vascular checks: risk assessment and management. Department of Health, London

9. Siu AL (2015) Screening for abnormal blood glucose and type 2 diabetes mellitus: U.S. preventive services task force recommendation statement. Ann Intern Med 163:861-868
10. Simmons RK, Echouffo-Tcheugui JB, Sharp SJ et al (2012) Screening for type 2 diabetes and population mortality over 10 years (ADDITION-Cambridge): a cluster-randomised controlled trial. Lancet 380:1741-1748

11. Simmons RK, Griffin, S.J., Witte, D.R., Borch-Johnsen, K., Lauritzen, T., Sandbæk, A. (2016) Effect of population screening for cardiovascular risk factors and type 2 diabetes on mortality and cardiovascular events among 1,912,392 Danish adults. Diabetologia DOI:10.1007/ s00125-017-4323-2

12. Griffin SJ, Borch-Johnsen K, Davies MJ et al (2011) Effect of early intensive multifactorial therapy on 5-year cardiovascular outcomes in individuals with type 2 diabetes detected by screening (ADDITIONEurope): a cluster-randomised trial. Lancet 378:156-167

13. Engelgau MM, Narayan KM, Herman WH (2000) Screening for type 2 diabetes. Diabetes Care 23:1563-1580

14. Lauritzen T, Griffin S, Borch-Johnsen K, Wareham NJ, Wolffenbuttel BH, Rutten G (2000) The ADDITION study: proposed trial of the cost-effectiveness of an intensive multifactorial intervention on morbidity and mortality among people with type 2 diabetes detected by screening. Int J Obes Relat Metab Disord 24(Suppl 3):S6-11

15. Christensen JO, Sandbaek A, Lauritzen T, Borch-Johnsen K (2004) Population-based stepwise screening for unrecognised type 2 diabetes is ineffective in general practice despite reliable algorithms. Diabetologia 47:1566-1573

16. Glumer C, Carstensen B, Sandbaek A, Lauritzen T, Jorgensen T, Borch-Johnsen K (2004) A Danish diabetes risk score for targeted screening: the Inter99 study. Diabetes Care 27:727-733

17. Conroy RM, Pyorala K, Fitzgerald AP et al (2003) Estimation of ten-year risk of fatal cardiovascular disease in Europe: the SCORE project. Eur Heart J 24:987-1003

18. Alberti KG, Zimmet PZ (1998) Definition, diagnosis and classification of diabetes mellitus and its complications. Part 1: diagnosis and classification of diabetes mellitus provisional report of a WHO consultation. Diabet Med 15:539-553

19. Green A, Sortso C, Jensen PB, Emneus M (2015) Validation of the Danish national diabetes register. Clin Epidemiol 7:5-15

20. UNESCO (1997) International Standard Classification of Education. Available from http://www.unesco.org/education/ information/nfsunesco/doc/isced 1997.htm. Accessed 20 Apr 2017

21. Huber PJ (1967) The behavior of maximum likelihood estimates under nonstandard conditions. In: Proceedings of the Fifth Berkeley Symposium on Mathematical Statistics and Probability. University of California Press, Berkeley, pp 221-233

22. Harris MI, Klein R, Welborn TA, Knuiman MW (1992) Onset of NIDDM occurs at least 4-7 yr before clinical diagnosis. Diabetes Care 15:815-819

23. Rahman M, Simmons RK, Hennings SH, Wareham NJ, Griffin SJ (2011) How much does screening bring forward the diagnosis of diabetes and reduce complications? 12-year follow-up of the Ely cohort. Diabetologia 55:1651-1659

24. Porta M, Curletto G, Cipullo D et al (2014) Estimating the delay between onset and diagnosis of type 2 diabetes from the time course of retinopathy prevalence. Diabetes Care 37:1668-1674

25. Danish National Health Service survey (2004). Available from https://www.sst.dk/da/sundhed-og-livsstil/tobak/tal-og-fakta/ danskernes-rygevaner/2004. Accessed 20 Apr 2017

26. Safwat S, Ishak RA, Hathout RM, Mortada ND (2017) Statins anticancer targeted delivery systems: re-purposing an old molecule. J Pharm Pharmacol. doi:10.1111/jphp.12707

27. Prasad V, Lenzer J, Newman DH (2016) Why cancer screening has never been shown to "save lives"-and what we can do about it. BMJ 352:h6080

28. Carstensen B, Kristensen J, Ottosen P, Borch-Johnsen K (2008) The Danish National Diabetes Register: trends in incidence, prevalence and mortality. Diabetologia 51:2187-2196 